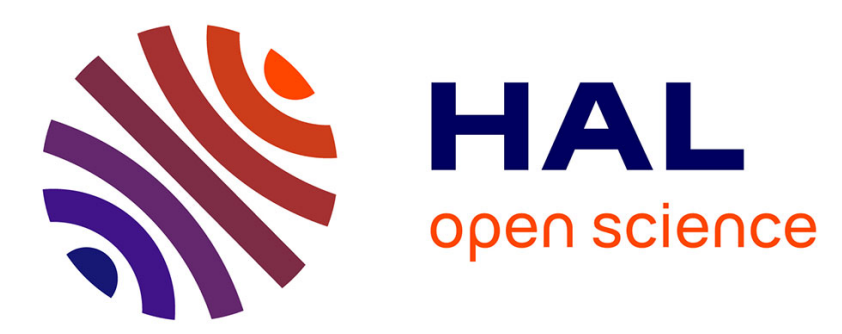

\title{
Mise en place du système racinaire du maïs. II. - Importance de quelques paramètres relatifs aux conditions de milieu
}

Marie-Odile Jordan

\section{To cite this version:}

Marie-Odile Jordan. Mise en place du système racinaire du maïs. II. - Importance de quelques paramètres relatifs aux conditions de milieu. Agronomie, 1987, 7 (7), pp.457-465. hal-00885015

\section{HAL Id: hal-00885015 https://hal.science/hal-00885015}

Submitted on 1 Jan 1987

HAL is a multi-disciplinary open access archive for the deposit and dissemination of scientific research documents, whether they are published or not. The documents may come from teaching and research institutions in France or abroad, or from public or private research centers.
L'archive ouverte pluridisciplinaire HAL, est destinée au dépôt et à la diffusion de documents scientifiques de niveau recherche, publiés ou non, émanant des établissements d'enseignement et de recherche français ou étrangers, des laboratoires publics ou privés. 


\title{
Mise en place du système racinaire du maïs. II. - Importance de quelques paramètres relatifs aux conditions de milieu
}

\author{
Marie-Odile JORDAN \\ I.N.R.A., Station d'Agronomie, Centre de Recherches de Colmar, B.P. 507, F-68021 Colmar
}

RÉSUMÉ

\begin{abstract}
Ce travail a pour objet de décrire l'influence des conditions de milieu (facteurs climatiques, édaphiques et trophiques) sur la mise en place du système racinaire du maïs.

Les paramètres descriptifs du développement dépendent très fortement de la plante, alors que ceux liés à la croissance subissent l'influence prépondérante des conditions de milieu. Les nombres d'organes, notamment des racines primaires, conserveront une certaine stabilité d'une situation pédo-climatique à l'autre. Les biomasses racinaires subissent de très fortes variations en fonction de l'humidité du substrat, du niveau de nutriments disponible, de la température, du peuplement...

Cependant, l'état de nos connaissances en ce qui concerne la structure racinaire, reste très limité. Ceci est imputable en partie, au manque de moyens appropriés, et au fait que les travaux effectués en ce domaine ont souvent été très fragmentaires, se limitant à l'étude de l'action d'un seul facteur constitutif du complexe climat-sol-plante, sur un seul paramètre descriptif de l'enracinement. Une approche plus globale, où l'on considérera le système racinaire dans sa totalité s'avère donc nécessaire.
\end{abstract}

Mots clés additionnels : Croissance, humidité, température, éléments nutritifs, structure de la racine.

This work deals with the influence of some environmental conditions (climate, soil moisture, and nutrient levels) on the growth and development of the corn root system. The specific parameters of development depend mainly on the plant, while those describing the growth of the root system are influenced by environmental conditions. So, the number of organs, especially the number of primary roots is constant at all experimental sites, while climate, soil moisture, nutrient level, and density plant have great influence on root dry matter. Our knowledge on this topic is rather limited. This is due to the lack of convenient technique. Most commonly the influence of only one factor of the climate-soil-plant complex on a single root parameter has been studied. A more global approach, in which the root system is considered as a whole, is necessary.

Additional key words : Growth, humidity, temperature, nutrients, root structure.

\section{INTRODUCTION}

L'enracinement d'une plante à un instant donné est fonction d'une double interaction entre la croissance et le développement du système racinaire, la croissance et le développement du système aérien d'une part et les conditions pédo-climatiques d'autre part.

$\mathrm{Au}$ cours d'une première étude bibliographique (JORDAN, 1987), nous nous sommes attachés à faire le point de nos connaissances sur les interactions entre les cinétiques de mise en place des appareils racinaire et aérien. Nous avons également évoqué les limites auxquelles se heurte l'étude de la structure racinaire.
Nous nous proposons ici de compléter ce travail en exposant les principaux résultats concernant l'influence du complexe climat-sol sur la mise en place du système racinaire du maïs. En effet, les rapports d'équilibres existant entre les différents organes d'une même plante sont susceptibles d'être modifiés par les conditions de milieu agissant sur l'ensemble du végétal. Ce que nous savons de l'influence des facteurs propres à la plante, et qui a été exposé antérieurement, doit être nuancé en fonction de l'hétérogénéité des conditions de culture.

Enfin, nous évoquerons les problèmes posés par l'intégration de ces données dans un modèle de struc- 
ture cohérent, l'importance d'un tel modèle pour l'étude du fonctionnement racinaire ayant déjà été évoquée (JORDAN, 1987). Il en est de même en ce qui concerne l'organisation du système racinaire du maïs et les problèmes posés par une telle étude.

\section{RÉGULATION DE LA CROISSANCE ET DU DÉVELOPPEMENT DE L'ENSEMBLE DU SYSTEMME RACINAIRE. INFLUENCE DES FACTEURS DU MILIEU}

Les facteurs de milieu peuvent être divisés en 3 catégories : ceux relatifs au climat, ceux liés au substrat, et ceux dépendants du peuplement. Les accidents végétatifs liés à des attaques parasitaires et à des toxicités alimentaires ainsi que les problèmes de mycorhization ne seront pas évoqués ici.

\section{A. Le climat}

Il intervient par l'intermédiaire de plusieurs de ses composantes.

\section{La température}

La température du sol est déterminante pour la croissance et le développement tant que le point végétatif de la plante est situé dans le sol, soit jusqu'à l'initiation de la 6 e feuille (PUJOS, 1975 ; WARRINGTON \& KANEMASU, 1983). Puis le métabolisme et la photosynthèse, la croissance et le développement du système aérien et dans une moindre mesure le système racinaire vont être fonction de la température de l'air. D'après RUSSELLE et al. (1984), celle-ci permettrait d'expliquer 95 p. 100 des variations de croissance et développement observées au champ.

CORNILLON (1974) a montré l'existence, chez la tomate, d'une température du sol, seuil, en deçà de laquelle il n'y a pas de croissance racinaire. L'effet positif du paillage ou de la pose de bâches plastiques mis en évidence pour diverses cultures, dont le maïs (ARMY et al., 1960 ; ADAMS, 1962 ; BANSAL et al., 1971) s'expliquerait en partie par le fait que ce seuil serait plus vite dépassé au printemps.

La température des racines fait varier la valeur du rapport PSA/PSR* (COOPER, 1973). Curieusement, pour le maïs, ce rapport baisse avec la température jusqu'à $15^{\circ} \mathrm{C}$ pour augmenter ensuite alors que chez la plupart des graminées des pays tempérés, on observe une hausse pouvant ou non, être suivie d'une baisse. Une augmentation de cette température fait également baisser le nombre de poils racinaires et le diamètre des racines (COOPER, 1973).

Enfin, le tropisme est fortement affecté. D'une façon générale, en conditions contrôlées, les racines sont d'autant plus verticales que la température est élevée, et ce, dans des limites comprises entre 10 et $27^{\circ}$ pour le soja (KASPAR et al., 1981) et 17 à $36^{\circ}$ pour le maïs (MOSHER \& MiLLER, 1972). Dans ce dernier cas, l'angle A, formé entre les racines primaires

\footnotetext{
* PSA/PSR : Poids sec aérien, poids sec racinaire.
}

(rattachées à la tige ou à la graine) et la verticale varie de 61 à $30^{\circ}$ (SHEPPARD \& MiLleR, 1977). ONDERDONK \& KETCHESON (1973) définissent la relation existant entre les 2 facteurs, comme suit : soit D la distance du point considéré au centre du grain (D restant inférieur à $15 \mathrm{~cm}$ ), $\mathrm{T}$ la température maximale journalière appliquée au sol en degrés $\mathrm{C}$ :

$\mathrm{A}=2393,46-1680 / \mathrm{T}+0,02721 \mathrm{~T}^{3}-0,0006105 \mathrm{~T}^{4}$ $-7,960 \mathrm{D}^{1 / 8}+0,49 \mathrm{D}^{2}$

Par contre, les gradients thermiques existant dans un même sol semblent n'avoir aucune influence sur le thermotropisme (SHEPPARD \& MILLER, 1977).

La température de l'air agit sur le nombre de racines secondaires ; la ramification maximale étant obtenue à $20^{\circ}$ pour le maïs et à $34^{\circ}$ pour le pois (NYE \& TINKER, 1977). D'autre part, le rapport entre les matières sèches aériennes (MSA) et racinaires (MSR) augmente linéairement quand la moyenne journalière passe de 15 à $28{ }^{\circ} \mathrm{C}$ (BRouwer, 1967) voire à $33{ }^{\circ} \mathrm{C}$ (ANDERSON \& KEMPER, 1964 ; WALKER, 1969) pour le maïs. Pour cette même plante, ces résultats confirment ceux donnés par COOPER (1973) pour la température racinaire, et sont dus au fait qu'un réchauffement provoque une augmentation des vitesses de croissance plus importante pour les parties aériennes que pour les parties racinaires (GROBBELAAR, 1963). Toujours chez le maïs, un développement optimal est obtenu entre 25 et $27^{\circ}$ (GROBBELAAR, 1963 ; BROUWER \& de WITT, 1969, WALKER, 1969) alors que ce seuil se situe à $27^{\circ}$ pour le pois et à $32^{\circ}$ pour le coton (ARNDT, 1945 ; GERARD, 1971).

\section{L'éclairement}

En limitant la photosynthèse et le transfert d'hydrates de carbone vers les racines, le manque de lumière affecte fortement la croissance racinaire et la valeur du rapport MSA/MSR qui va diminuer après une première phase d'augmentation (LAMBERT \& BAKES, 1979). D'autre part, ces mêmes auteurs relèvent une augmentation de la concentration d'acide abscissique et de cytokinine, tous deux inhibiteurs de croissance, dans les apex racinaires. Les vitesses d'élongation ainsi que les taux d'apparition de racines secondaires sont réduits (LAMBERS \& POSTHUMUS, 1980 ; LAMBERS, 1983).

\section{Relations entre l'eau dans le sol et la plante}

L'état hydrique du sol agit sur le végétal en régulant la transpiration et l'ouverture stomatique. De plus, il est susceptible de modifier certains paramètres descriptifs de la structure et de la cinétique racinaire, en particulier : les rythmes d'élongation, le nombre de secondaires et les diamètres ainsi que la densité racinaire dans les différents horizons.

\section{a) L'élongation racinaire}

Il a été montré en conditions artificielles que, chez le maïs, l'élongation racinaire est une fonction linéaire décroissante du potentiel hydrique du sol, l'augmentation de celui-ci induisant un stress hydrique et osmotique et une réduction de l'activité enzymatique (GiNGRICH \& RUSSEL, 1956 et 1957 ; GERARD, 1971). En 
outre, en chambre de culture, la croissance racinaire du blé est proportionnelle à l'ETP (définie par PENMAN, 1963 ; BROCHET \& GERBIER, 1974) en début du cycle (DERERA et al., 1969). Les causes physiologiques de cet état de fait ne sont pas encore connues.

\section{b) La densité racinaire dans les différents horizons}

La répartition du système dans les différents horizons ainsi que son importance relative sont directement fonction des réserves en eau du sol. STRUIK \& BRAY (1970, dans NYE \& TINKER, 1977) trouvent que, chez le maïs, l'appareil racinaire représente 33 p. 100 du poids sec en conditions sèches et 13 p. 100 si l'humidité du sol est non limitante. La valeur du rapport MSR/MSA pourrait subir des variations de 15 p. 100 en fonction de la teneur en eau du sol (MILLER, 1916). Toujours chez le maïs, on constate que l'enracinement est plus profond sur un sol sec. Une double régression englobant la profondeur et l'humidité explique 75 p. 100 des variations de densité racinaire observées (GRIMES et al., 1975) chez cette plante. Sous irrigation, 64 p. 100 des racines sont situés à moins de $30 \mathrm{~cm}$, et 99 p. 100 à moins de $90 \mathrm{~cm}$, alors que ces proportions sont respectivement de 39 et de 70 p. 100 en conditions non irriguées, les différences observées étant beaucoup moins nettes en ce qui concerne le sorgho et le soja (MAYAKI et al., 1976). L'ensemble de ces résultats est obtenu au champ ou en rhizotrons, et les chiffres avancés sont fonction, indépendamment de l'humidité du sol, de l'interaction effet variétal-conditions de milieu autres que l'état hydrique du sol. Ils confirment l'hypothèse couramment admise selon laquelle la croissance des organes susceptibles d'assurer l'alimentation de la plante en un élément déficient est favorisée ; les conditions de milieu peuvent agir sur les états d'inductions internes qui déterminent les potentiels de croissance des appareils aériens et racinaires (BEHAEGUE, 1979, 1981). Même si la réalisation du programme de croissance des parties aériennes est prioritaire (GILLET et al., 1984), il semblerait que celui-ci soit déterminé de façon à pouvoir assurer à la plante un enracinement optimal.

D'autre part, la colonisation racinaire est plus uniforme en conditions humides (WEAVER, 1926), auquel cas la résistance mécanique à la pénétration est beaucoup plus faible, et le sol, quelle que soit sa structure, plus perméable aux racines.

\section{c) Le nombre de secondaires}

La configuration spatiale du système racinaire peut également être modifiée par des variations d'humidité. C'est ainsi que, chez le maïs, une diminution de l'humidité du sol, induit (tant que l'on reste en conditions non limitantes) une augmentation du nombre de ramifications (WEAVER, 1926) et de la distance entre l'apex des primaires et la dernière ramification secondaire (LAMBERS \& POSTHUMUS, 1980).

\section{d) Le diamètre racinaire}

S'il y a manque d'eau, le chevelu disparaît et le diamètre racinaire est réduit. Sur de jeunes plantules, cette réduction est définitive et les racines s'atrophient si le dessèchement est durable (COUCHAT et al., 1980).
Cette réduction peut être observable uniquement durant les heures chaudes de la journée et peut atteindre 40 p. 100 chez le coton (HUCK et al., 1970), 60 p. 100 chez le blé (COLE \& Alston, 1974) et 30 p. $100 \mathrm{chez}$ le tournesol (FAIz et al., 1982). Elle est, dans ce dernier cas, reliée à l'augmentation de l'ETP et du potentiel hydrique $\mathrm{du}$ sol. En cas d'absorption hydrique insuffisante, les racines qui contiennent normalement 50 p. 100 de l'eau du végétal, assurent durant un certain temps de 60 à 70 p. 100 de la fourniture en eau du végétal. Durant cette phase, elles peuvent perdre jusqu'à 45 p. 100 de leur poids, ce qui explique la réduction importante du diamètre. En deçà d'un certain seuil de dessiccation, la réhumectation des tissus racinaires est possible et se fera au courant de la nuit (CRUIZIAT, 1974).

Une sécheresse prolongée provoque enfin des modifications physiologiques chez certaines dicotylédones (Crucifères, Papavéracées) et l'apparition de racines courtes, tubérisées à forte réserve en amidon. Le nombre de leurs cellules reste comparable à celui des ébauches normales, mais leur taille augmente sensiblement de façon à permettre une mise en réserve. Ces racines, initiées au moment de la fermeture stomatique constituent une réserve méristématique importante, permettant la reprise rapide de l'élongation racinaire après la réhumectation du substrat. Ce phénomène n'a pas pu être mis en évidence chez les monocotylédones (VARTANIAN, $1972 a$ et $b$ et 1981 ).

\section{B. Le sol}

Il complète l'action prépondérante du climat et agit par l'intermédiaire de ses propriétés physiques (agrégation et résistance mécanique à la pénétration) et chimiques (importance de ses bases échangeables). L'ensemble de ces phénomènes ayant déjà été décrit dans plusieurs ouvrages très complets (WIERSMA, 1959 ; SCOTT-RUSSEL, 1977 ; NYE \& TINKER, 1977 ; HARLEY \& SCOTT-RUSSEL, 1979), nous nous limiterons à une brève synthèse.

\section{Les propriétés physiques}

L'état structural, qui est sous la dépendance des techniques culturales, va déterminer la pression exercée sur les apex racinaires en croissance. D'autre part, il induit un état poral conditionnant la circulation de l'eau et des gaz et détermine en particulier la tension d'oxygène. Cette dernière est corrélée aux poids secs aériens et racinaires, voire au rendement (HAGIN, 1952 ; WIEGAND \& LEMON, 1958 ; PHILIPS \& KIRKHAM, 1962 ; ANDERSON \& KEMPER, 1964) chez le maïs et le sorgho, cultivés au champ et en containers. Ceci serait dû au fait, qu'en conditions d'aération faible, consécutives à une porosité insuffisante ou à un remplissage des pores par l'eau du sol, la respiration racinaire serait très fortement diminuée, ce qui aurait un effet dépressif sur le développement de l'ensemble de la plante. La valeur optimale de la tension d'oxygène serait de 0,22 atmosphère (NYE \& TINKER, 1977), et la valeur minimale de 0,10 atmosphère pour le maïs (LiNSCOTT et al., 1962). Cette tension est capable d'agir sur l'inclinaison racinaire (KASPAR et al., 1981) 
et est corrélée positivement à la croissance racinaire, ainsi qu'au poids sec du système chez le maïs (GINGRICH \& RUSSEL, 1956 et 1957). L'effet est moins marqué sur le blé, où le nombre de cellules formées augmente bien avec la teneur en oxygène du sol. Mais la taille de celles-ci se réduisant à mesure, l'effet sur le poids total est nul, voir négatif (BOSEMARK, 1954). D'autre part, l'éthylène, autre composante de l'atmosphère du sol, influe de façon positive sur les taux d'élongation (DREW \& GoSS, 1973).

La porosité a aussi une influence plus directe. En effet, pour assurer un développement racinaire optimal, il faut que les pores, occupés de façon préférentielle, présentent une certaine continuité entre les différents horizons. WIERSUM (1961, dans Goss et al., 1984) et WELBANK (1974, dans Goss et al., 1984) pensent que seulement 1 à 5 p. 100 des pores sont colonisés par les racines, mais que ce chiffre oscille entre 10 et 50 p. 100 en ce qui concerne ceux présentant une continuité avec les horizons profonds. Un effet écran entre les différents horizons a été mis en évidence par TARDIEU (1984), sur une culture de maiis au champ. En effet, un horizon profond, même très meuble, ne va pas être colonisé sous les zones tassées de l'horizon supérieur où les racines n'ont pas pu pénétrer. Paradoxalement, une augmentation de la densité racinaire va faire diminuer la porosité et la perméabilité du substrat (BARLEY, 1954).

D'autre part, le substrat oppose une résistance purement mécanique à la pénétration racinaire. D'une façon générale, celle-ci va être d'autant plus importante que la granulométrie est fine et le taux d'humidité résiduelle faible. Elle atteint sa valeur maximale pour un sol contenant moins de 18 p. 100 d'eau et plus de 40 p. 100 d'argile, la nature de celle-ci n'ayant aucune importance (MAERTENS, 1964).

La résistance à la pénétration n'affecte pas l'initiation racinaire (PICARD, 1976) mais affecte fortement la croissance, et ce, d'autant plus que les variations de densité sont brutales. Chez le maïs, la résistance à l'enfoncement du pénétromètre peut être corrélée aux vitesses d'élongation $\left(r=0,822^{* *}\right)$ (PHILIPS \& KIRKHAM, 1962 ; NYE \& TINKER, 1977). EAVIS (1972) et MIRREH \& KETCHESON (1972 et 1973) ont mis en évidence la relation suivante, où $Y$ est l'élongation racinaire, $X_{1}$ la résistance du sol à la pénétration et $X_{2}$ le potentiel matriciel en bars.

$$
\mathrm{Y}=\mathrm{A}+\mathrm{BX}_{1}+\mathrm{CX}_{2}+\mathrm{DX}_{1}^{2}+\mathrm{EX}_{2}^{2}+\mathrm{FX}_{1} \mathrm{X}_{2}
$$

$A, B, C, D, E, F$ sont fonction de la variété et du type de sol.

Toujours chez le maïs, la croissance est fortement ralentie si les pressions exercées par les particules de sol atteignent $3 \mathrm{~kg} / \mathrm{cm}^{2}$ pour l'ensemble du système racinaire et $10 \mathrm{~kg} / \mathrm{cm}^{2}$ si elles s'appliquent uniquement à l'apex. Des valeurs de l'ordre de 6 et de $12 \mathrm{~kg} / \mathrm{cm}^{2}$ dans chacun des cas ont pour effet de stopper toute élongation; le stress induit perturbe non seulement la croissance mais l'ensemble du métabolisme et il est d'autant plus important que les tissus sont âgés (BARLEY, 1962; BARLEY \& GREACEN, 1967). GILL \& MILLER (1956) ont mis en évidence un changement de la morphologie racinaire en fonction de l'augmentation de la pression exercée sur l'apex. Une compression de la zone d'élongation va réduire la taille du système, mais ne perturbe pas la division cel- lulaire. Toutefois, les cellules formées sont plus petites et leur pression osmotique plus importante, ce qui induit un diamètre racinaire plus important (FELDMANN, 1984).

\section{Les propriétés chimiques}

Outre son influence par l'intermédiaire de ses propriétés physiques, le sol constitue pour le végétal un réservoir d'éléments nutritifs dont le niveau de disponibilité marque fortement le développement racinaire.

Des expériences en solution nutritive ont montré qu'en conditions non restrictives, la longueur totale du système est d'autant plus importante que le niveau de dilution est faible (MAY et al., 1966). D'une façon générale, une carence faible ne modifie pas, ou fait baisser légèrement les taux d'élongation mais stimule fortement l'initiation des ramifications de tous ordres. Si elle se prolonge, la croissance baisse, puis s'arrête. Il peut même y avoir nécrose des apex racinaires (TENNANT, 1976 ; de JAGER, 1982).

L'azote a été l'élément le plus étudié (MAIZLISH et al., 1980 ; BROUWER et al., 1981 ; GEISLER \& KRUTZFELDT, 1983 ; LAMBERS, 1983). Le riz développe un enracinement d'autant plus profond que le niveau de fertilisation est faible, la matière sèche racinaire totale étant fonction de la dose d'azote épandue. Il en est de même pour le nombre d'ordres racinaires présents : on note la présence de racines d'ordre 3 si on applique uniquement une fumure au semis, et de racines d'ordre 5 si celle-ci est complétée par 2 épandages de surface (KAWATA et al., $1977 a$ et $b$ ). Chez le ray-grass, de fortes concentrations en azote stimulent également la ramification. De plus, la matière sèche aérienne et racinaire augmente avec la dose d'azote épandue pour atteindre un palier avant de décroître. Cependant, l'optimum est plus faible pour les parties racinaires que pour les parties aériennes. En fonction des doses d'azote croissantes, on distingue donc 3 cas de figure successifs : on observe tout d'abord un effet bénéfique pour l'ensemble de la plante, puis un effet dépressif pour les racines et bénéfique pour les parties aériennes et enfin un effet dépressif sur tout le végétal (LEMAIRE, 1975). Il est permis de supposer, que ces tonnées obtenues sur une plante pérenne sont également applicables aux graminées annuelles. Cela reste cependant à vérifier. D'autre part, une disponibilité correcte en azote augmente l'efficience de l'utilisation de l'eau par la plante chez le maïs (LinsCOTT et al., 1962). Les conséquences d'une carence sur le rendement sont fonction du stade où celle-ci est appliquée. Un stress appliqué à de jeunes plants de maïs entre le $18^{\mathrm{e}}$ et le $28^{\mathrm{e}}$ jour augmente le rapport MSR/MSA du $25^{\mathrm{e}}$ au $40^{\mathrm{e}}$ jour (GIRARDIN et al., 1985).

Une carence potassique induit directement une réduction de la surface, du poids sec et du volume racinaire (HACKETT, 1969) sans que l'on passe par une phase de latence.

Il peut exister des interactions positives entre 2 éléments, notamment entre le phosphore et l'azote (DUNCAN \& OHLROGGE, 1958). Le rôle des oligo-éléments n'a encore été que peu étudié.

L'absence d'un nutriment perturbe l'ensemble des phénomènes d'absorption du végétal et peut être plus 
ou moins invalidante suivant les conditions climatiques : température et photosynthèse (PITMAN, 1972 ; PETTERSON, 1975). Il est difficile de dissocier l'effet propre de chacun des métabolites, qui peut intervenir de façon complexe par l'intermédiaire de substances de croissance (cytokinine en ce qui concerne l'azote) (LAMBERS, 1983) et affecte non seulement le système racinaire, mais le végétal dans son ensemble. Une carence minérale pénalise d'abord le système aérien, alors qu'une concentration excessive semble avoir un effet inverse (LEMAIRE, 1975).

\section{Le peuplement}

Deux pieds voisins étant en compétition pour la colonisation du sol et l'absorption minérale comme ils le sont pour la photosynthèse, la densité du peuplement va influer sur la mise en place du système racinaire. Pour le blé et le maïs, l'évolution dans le temps des principaux paramètres descriptifs de la structure du système aérien : poids sec, hauteur, nombre de feuilles et de talles, surface foliaire, suit une loi statistique normale. Une certaine hétérogénéité entre les plantes apparaît dès la levée. Elle va être conservée tout au long du cycle, et les écarts observés entre les plantes vont se creuser (GLENN \& DAYNARD, 1974 sur maiis; MASLE-MEYNARD \& SEBILLOTTE, 1981 sur blé). On peut supposer qu'il existe des variations du même ordre pour le système racinaire. Cette hypothèse semble confirmée par les résultats de PICARD et al. (1985), qui trouvent, que chez le mais, la variabilité du nombre de racines par entrenœud augmente avec le rang de ce dernier. Or, chez cette plante, l'émission sur un entrenœud donné se fait d'autant plus tardivement que son rang est élevé et l'hétérogénéité stadiale augmente donc au cours du cycle.

D'autre part, si les individus isolés ont tendance à coloniser un volume de sol plus important et moins profond que s'ils étaient intégrés à un peuplement (RAPER \& BARBER, $1970 a$ et $b$ ), les densités de semis usuellement appliquées modifient peu la densité racinaire du sol. Evaluée par carottage (GRIMEs et al., 1975) (résultats sur coton et maîs), la longueur du système racinaire par plante décroît en proportion et cette décroissance peut atteindre 83 à 99 p. 100 selon les espèces (PAVLYCHENKO, 1937) (résultats obtenus sur blé, orge, seigle et folle-avoine). Une augmentation de la densité, en limitant le volume de sol disponible pour chaque plante, va non seulement affecter le système racinaire, mais limite également, pour chaque plante, le développement des parties aériennes comme le montrent les expérimentations de PETTERSON et al. (1984) portant du blé en conteneurs de tailles diverses.

De plus, dans le cas des graminées, les systèmes racinaires de deux plantes voisines peuvent s'interpénétrer (PAVLYCHENKO, 1937 - étude sur blé, orge, seigle ; NYE \& TINKER, 1977).

Enfin, les espèces favorisées en ce qui concerne la compétition interspécifique sont celles dont la mise en place du système racinaire est la plus rapide et dont l'enracinement est régulier le long du profil (PARRISH \& BAZZAZ, 1976).

D'une manière générale, le peuplement influe plus sur la croissance que sur le développement.

\section{Conclusion}

La démarche adoptée dans la plupart des études présentées ici consiste à expliquer l'évolution d'un seul paramètre descriptif de l'enracinement en fonction d'un seul facteur de milieu. Les méthodes utilisées se heurtent à des biais spécifiques. En conditions contrôlées on utilise un substrat artificiel ou un sol remanié avec une température et un éclairement souvent constants. Les résultats ainsi obtenus peuvent servir de références pour l'estimation de l'évolution du potentiel racinaire en fonction du facteur étudié, mais ils ne sont pas directement transposables en conditions de grande culture (KLEPPER et al., 1983 ; KOPKE, 1981). D'autre part, dans les expérimentations de terrain, le nombre de facteurs tant internes qu'externes à la plante qui interviennent dans la régulation de la croissance et du développement racinaire, la complexité de leur mode d'action et d'interaction les uns par rapport aux autres, rendent difficile la description de l'influence exacte d'un seul facteur de milieu sur la cinétique du système racinaire. Enfin, les problèmes méthodologiques concernant la description objective et quantitative de l'état structural du profil ne permettent souvent pas de généraliser les résultats obtenus, et peuvent expliquer en partie les incohérences mises en évidence dans cette revue.

Pour progresser dans la connaissance du double système d'interactions entre la croissance et le développement des parties racinaires, la croissance et le développement du système aérien d'une part, et les conditions pédo-climatiques d'autre part, dont dépend l'enracinement d'une plante à un instant donné, ces études très ponctuelles doivent être complétées par une approche plus globale.

La modélisation constitue un outil privilégié pour ce type de travail (PRASAD et al., 1983).

\section{ESSAI DE MODÉLISATION DES CONNAISSANCES ACQUISES}

En général, l'étude d'un phénomène intéressant l'agronome passe par l'élaboration successive de 2 types de modèles (PICARD et al., 1985). Ceux-ci seront tout d'abord déterministes et auront une valeur explicative mais non prédictive car les phénomènes aléatoires ne seront pas pris en compte. Dans un second temps, on aboutira à des modèles probabilistes qui auront un rôle plus prédictif.

Il faudra donc, dans un premier temps, construire un modèle architectural du système racinaire du maïs, permettant de savoir, quand et comment le système racinaire se met en place dans les conditions de culture les plus fréquemment rencontrées. Au cours de cette $1^{\text {re }}$ phase, il importe de dégager les invariants liés à l'espèce et à la variété, donc constants quelles que soient les conditions de milieu.

Ce modèle, comparé à des situations réellement observées au champ, pourra ensuite servir de référence dans l'étude de la variabilité de la structure du système racinaire induite par les conditions pédoclimatiques. C'est au cours de cette seconde phase que seront intégrés au modèle, les résultats concernant 
l'action et les interactions des facteurs de milieu sur la cinétique racinaire. On aboutira ainsi à un modèle probabiliste permettant de décrire, a priori, la cinétique racinaire du maïs.

Ce dernier modèle pourra ensuite être intégré aux modèles d'absorption d'eau et d'éléments nutritifs déjà évoqués en introduction afin d'augmenter leur précision et leur fiabilité.

La $1^{\text {re }}$ étape du travail a été abordée par plusieurs auteurs qui ont essayé d'élaborer des modèles de structure simple, décrivant l'évolution at cours du cycle, d'un seul, voire de 2 paramètres descriptifs. L'évaluation effectuée est, soit purement théorique, soit fondée sur des mesures de terrain, rarement suffisantes pour permettre l'étude de tous les facteurs intervenants. On peut distinguer :

- les modèles de croissance où la production de matière sèche est estimée à partir du niveau de photosynthèse. Pour PENNING de VRIES et al. (1974), 21 p. $100 \mathrm{du}$ glucose synthétisé sert à la maintenance et 79 p. 100 à la construction de molécules de structure. On a, dans un $1^{\text {er }}$ temps, étudié la répartition des assimilats carbonés et azotés entre les parties aériennes et racinaires (BROUWER \& de WITT, 1969; THORNLEY, 1972 ; THORNLEY, 1976). Les concepts énoncés ont ensuite été appliqués aux cultures de plein champ par HUCK \& HILLEL (1983) dans le cas d'un peuplement végétal et par COELHO \& DALE (1980) et GIRARDIN (1982) dans le cas du maiis ;

- les modèles de structure où l'on s'attachera à suivre l'évolution du nombre d'organes et de leur disposition spatiale. On distinguera d'une part les travaux purement architecturaux comme ceux de KAHN (1977 et 1978), qui construit un modèle en 3 dimensions des systèmes racinaires des arbres tropicaux, où l'orientation de chaque racine est fonction de l'ordre auquel elle appartient et de son niveau d'insertion sur la racine porteuse. Cette étude s'inspire du modèle théorique de FRANQUIN (1970, 1972) sur la structure des parties aériennes. D'autre part, de nombreux travaux sont fondés sur les rythmes d'émission des racines primaires en fonction de leur niveau d'insertion et du nombre de feuilles totales de la plante. Dans le modèle de KLEPPER et al. (1984), l'observation d'un grand nombre de pieds de blé a permis d'établir statistiquement la cinétique d'apparition des racines. Cette étude qui complète les travaux de KIESSELBACH (1949) sur le maïs, de FRIEND (1965) sur le blé et de GOUNOT et al. (1980) sur le dactyle, décrit la structure des graminées. Selon ces auteurs celles-ci seraient composées d'unités identiques et superposées ;

- les modèles de synthèse où interviennent à la fois des paramètres descriptifs de la croissance et de la structure racinaire. Le modèle de structure du système racinaire des graminées construit par LUNGLEY (1973) se rattache à cette catégorie. Il est construit à partir de 8 paramètres : le rythme d'émission des racines primaires, les vitesses d'allongement des racines primaires, secondaires et tertiaires, la distance entre l'extrémité apicale et la dernière ramification des primaires et secondaires ainsi que leurs rythmes de ramification.

Ces paramètres, fonction de facteurs pédo-climatiques comme la température et l'humidité du substrat, permettent de suivre l'évolution au cours du temps des densités racinaires dans le sol (HACKETT \& ROSE,
$1971 a$ et $b$; HACKETT, 1973 ; GERWITZ \& PAGE, 1974 ; LAMBERT \& BAKES, 1979 ; NARDA \& CURRY, 1981).

La plupart des travaux présentés ici procèdent d'une approche descriptive en ce qui concerne l'étude de l'établissement de la structure racinaire. Cependant, le modèle de WANG (1983), établi pour de jeunes plantules de maïs dont les racines n'excèdent pas $10 \mathrm{~cm}$, et fondé sur l'évolution des temps de germination, de l'orientation racinaire et des vitesses de croissance en fonction de la résistance à la pénétration, de la concentration en oxygène, de la température et du potentiel matriciel du sol, a une valeur prédictive.

Il importe maintenant d'étendre ce type de travail à l'ensemble du cycle cultural de la plante. Mais il faudra auparavant :

- compléter nos connaissances. Si le comportement des racines primaires (rythme d'émission, élongation, ramification et orientation) commence à être bien connu, il n'en est pas de même en ce qui concerne les racines secondaires et surtout tertiaires. Les quelques données dont nous disposons sont obtenues en conditions artificielles (cultures hydroponiques ou containers) et sont donc difficilement transposables au champ (KOPKE et al., 1982 ; KLEPPER et al., 1983) ;

- pouvoir quantifier les relations existant entre la structure du sol, sa variation au sein du volume colonisé par une plante d'une part, et la mise en place du système racinaire, d'autre part. En raison de problèmes méthodologiques, très peu de données sont disponibles en ce domaine (TARDIEU, 1984).

Cette étape une fois franchie, les modèles de structure racinaire pourront être intégrés aux modèles d'absorption à l'exemple du modèle SOYROOT concernant le soja et qui permet d'évaluer l'alimentation hydrique du végétal (NARDA \& CURRY, 1981). Les niveaux d'absorption pourront alors être déterminés, non pas en fonction de ce qui est disponible dans le sol, mais en fonction de ce qui est absorbable par les racines, compte tenu de la migration très faible dans le sol de certains éléments comme le phosphore ou la potasse, et de la distance entre les racines et chaque point du profil.

\section{CONCLUSION}

La cinétique de croissance et de développement du système racinaire du maîs est soumise à des phénomènes de régulation complexes, jouant à plusieurs niveaux. Dans cette étude, nous nous sommes intéressés uniquement aux modifications induites par le milieu sur les interactions entre les différents organes qui se développent de façon harmonieuse les uns vis-àvis des autres. L'importance des facteurs internes à la plante a fait l'objet d'un travail antérieur (JORDAN, 1987).

Les conclusions établies au cours de ce premier travail restent valables.

Le fait que les paramètres constitutifs du développement sont moins sensibles aux variations du milieu que ceux relatifs à la croissance semble confirmé.

L'enracinement ayant surtout été étudié en fonction 
du complexe climat-sol, nous disposions d'un nombre de références très important pour le second volet de cette recherche bibliographique par rapport à la $1^{\text {re }}$ partie de ce travail.

Cette revue montre que nos connaissances en ce domaine restent très fragmentaires en raison notamment de problèmes méthodologiques.

La première difficulté réside dans le choix des paramètres à étudier. Peu de travaux ont essayé de définir la structure racinaire et les paramètres qui permettent de la décrire précisément. Même le travail de synthèse de LUNGLEY (1973) est incomplet à cet égard car il ne mentionne pas l'orientation des racines.

De plus, les méthodes à mettre en œuvre pour la caractérisation de l'enracinement (SCHURMAN \& GOEDEWAAGEN, 1971 ; BÖHM, 1979) sont très coûteuses en temps (KOPKE, 1981 ; PICARD, 1984), et ne permettent souvent pas de suivre la cinétique de croissance et de ramification en profondeur. De nouvelles techniques sont actuellement à l'étude pour pallier à ces inconvénients (MAERTENS \& ClAUzel, 1982; MAERTENS \& PICARD, 1984).

Enfin, les résultats obtenus par diverses méthodes sont contradictoires (KOPKE, 1981), ce qui pose des problèmes pour la généralisation des résultats obtenus.

Notre connaissance très limitée de la structure du système racinaire est également due à la façon dont s'est faite cette investigation. En effet, « 3 types de démarches sont à mettre en ouvre simultanément pour l'étude d'un phénomène intéressant l'agronome " (SebillotTe, 1974). Il faut :

- approfondir notre connaissance des relations sol- climat-plante et mieux comprendre comment elles expliquent le problème étudié,

- faire une analyse descriptive de la situation « in situ » et interpréter la gamme de variations observée,

- juger de l'importance d'un paramètre à l'aide d'une étude fréquentielle.

Une analyse des travaux présentés (notamment des modèles) montre que la $1^{\text {re }}$ démarche a été très fréquemment mise en œuvre, alors que les études globales de terrain ont été peu employées. Par conséquent, si nous savons comment va influer un facteur sur un paramètre descriptif de l'enracinement, notre connaissance du rôle de l'interaction de ces mêmes paramètres entre eux, prépondérante au champ, est relativement limitée.

Une meilleure connaissance de la structure du système racinaire permettrait :

- au chercheur d'améliorer ses connaissances concernant le fonctionnement du système racinaire, par exemple de relier les niveaux d'absorption des éléments nutritifs au diamètre des racines (LESAINT et $a l ., 1979)$ et donc dans le cas du maïs à un entrenœud donné. Ceci permettrait notamment de mieux raisonner la fertilisation en effectuant les apports après l'émission racinaire sur les entrenœuds considérés ;

- au sélectionneur de créer des variétés résistantes à la verse racinaire qui dépendrait du nombre de racines adventives et de leur angle d'insertion sur le pied (PINTHUS, 1969 ; ZUBER, 1968) ;

- à l'agriculteur d'ajuster sa fertilisation donc de réduire ses charges de culture.

Reçu le 11 juin 1986. Accepté le 21 mars 1987.

\section{RÉFÉRENCES BIBLIOGRAPHIQUES}

Adams J. E., 1962. Effect of soil temperature on grain sorghum growth and yield. Agron. J., 54, 257-261.

Anderson W.-B., Kemper W.-D., 1964. Corn growth as affected by aggregate stability, soil temperature, soil moisture. Agron. J., 56 (5), 453-456.

Army T. J., Elmer B., Hudspeth J., 1960. Alteration of the microclimate of the seed zone. Agron. J., 52 (12), 17-22.

Arndt C. H., 1945. Temperature-growth relations of the roots and hypocotyls of cotton seedlings. Plant Physiol., 2, 200-220.

Bansal S.-P., Gajri P. R., Priar S.-S., 1971. Effect of mulches on water conservation, soil temperature and growth of maize (Zea mays L.) and pearl millet (Pennisetum typhoides). Indian J. agric. Sci., 41 (5), 467-473.

Barley K. P., 1954. Effects of root growth and decay on the permeability of a synthetic sandy loam. Soil Sci., 78, 205-210.

Barley K. P., 1962. The effect of mechanical stress on the growth of roots. J. exp. Bot., 13 (37), 97-110.

Barley K. P., Greacen E. L., 1967. Mechanical resistance of a soil influencing the growth of roots and underground shoots. $A d v$. Agron., 19, 1-43.

Behaegue T. J., 1979. Eco-fysiologische grondslagen van de grasgroi. Agricultura, Louvain, 23 (4), 375-392.

Behaegue T. J., 1981. Exploitation des prairies. 4. La variation dans la croissance de l'herbe ; causes et conséquences pour l'exploitation des prairies. Agricultura, Louvain, 34 (3), 511-515.

Böhm W., 1979. Methods of studying root systems. Ecological studies 33, Springer Verlag, $188 \mathrm{p}$.
Bosemark N. O., 1954. The influence of nitrogen on root development. Physiol. Plant., 7, 497-502.

Brochet P., Gerbier M., 1974. L'évapotranspiration. Aspects agrométéorologiques. Monographie 65. Météo Nationale, Paris, 95 p.

Brouwer R., 1967. Beziehungen zwischen Spross- und Wurzelwachstum. Angew. Bot., 41, 244-254.

Brouwer R., de Witt C. T., 1969. A simulation model of plant growth with special attention to root growth and its consequences. In : "Root Growth», ed. Whitringion W. J., Butterworths, London, 224-244.

Brouwer R., Gasparikowa O., Kolek J., Loughman B. C., 1981. Structure and function of plant roots. Proceedings of the 2nd Symposium held in Bratislava, Czechoslovakia, Sept. 1-5, 1980. Martinus Nijhoff, London.

Coelho D. T., Dale R. F., 1980. An energy-crop growth variable and temperature function for predicting corn growth and development : planting to silking. Agron J., 72, 503-510.

Cole P., Alston A. M., 1974. Effect of transient dehydration on absorption of chloride by wheat roots. Plant Soil, 40, 243-247.

Cooper A. J., 1973. Root temperature and plant growth. Commonwealth Agricultural Bureaux, Slough, England, 73 p.

Cornillon P., 1974. Comportement de la tomate en fonction de la température du substrat. Ann. agron., 25 (5), 753-777.

Couchat Ph., Moutonnet P., Houelle M., Picard D., 1980. In situ study of corn seedlings root and shoot growth by neutron radiography. Agron. J., 72, 321-324. 
Cruiziat P., 1974. Détermination des pertes en eau subies par les différents organes d'une plante soumise au dessèchement. Ann. agron., 25 (4), 539-554.

Derera N. F., Marshall D. R., Balaam L. N., 1969. Genetic variability in root development in relation to drought tolerance in spring wheats. Exp. Agric.,5, 327-337.

Drew M. C., Goss M. J., 1973. Effect of soil physical factors on root growth. Chem. Ind., 21, 679-684.

Duncan W. G., Ohlrogge A. J., 1958. Principles of nutrient uplake from fertilizer bands. 11. Root development in the bands. Agron. J., 10, 605-608.

Eavis B. W., 1972. Soil physical conditions affecting seedling root growth. 1. Mechanical impedance, aeration and moisture availability as influenced by bulk density and moisture levels in a sandy loam soil. Plant Soil, 36, 613-622.

Faiz S. M. A., Weatherley P. E., 1982. Root contraction in transpiring plants. New Phytol., 92, 333-343.

Feldmann L. J., 1984. Regulation of root development. Annual. Rev. Plant Physiol., 35, 223-242.

Franquin P., 1970. Modèles mathématiques de structure chez les végétaux. 1. Cah. ORSTOM, Ser. Biol., n ${ }^{\circ}$ 14, 77-125.

Franquin P., 1972. Modèles mathématiques de structure chez les végétaux. II. Cah. ORSTOM, Ser. Biol., n ${ }^{\circ}$ 17, 3-21.

Friend D. J. C., 1965. Tillering and leaf production in wheat as affected by temperature and light intensity. Can. J. Bot., 43, 1063 1076.

Geisler G. N., Krutzfeldt B., 1983. Investigations into the effect of "nitrogen" on the morphology, dry matter formation and nutrient uptake efficiency of the root systems of maize, spring barley and field bean varieties, having regard to temperature conditions. I. Root morphology. Z. Acker Pflanzenbau, 152 (5), 336-353.

Gerard C.-J., 1971. Influence of osmotic potential, temperature and calcium on growth of plant roots. Agron. J., 63, 555-558.

Gerwitz A., Page E. R., 1974. An empirical mathematical model to describe plant root systems. J. appl. Ecol., 11, 773-782.

Gill W.-R., Miller R.-D., 1956. A method for study of the influence of mechanical impedance and aeration on the growth of seedling roots. Soil Sci. Soc. Am. Proc., 20, 154-157.

Gillet M., Lemaire G., Gosse G., 1984. Essai d'élaboration d'un schéma global de la croissance des graminées fourragères. Agronomie, 4 (1), 75-82.

Gingrich J. R., Russell M. B., 1956. Effect of soil moisture tension and oxygen concentration on the growth of corn roots. Agron. J., 48, 517-520.

Gingrich J. R., Russell M. B., 1957. A comparison of effects in soil moisture tension and osmotic stress on root growth. Soil Sci, 84 185-194.

Girardin Ph., 1982. Essais de modélisation de la croissance et du développement du mais grain. Thèse, Doct. Ing. Université de Paris-Sud, Ecologie Végétale, 111 p.

Girardin Ph., Tollenaar M., Muldoon J. F., 1985. Effect of temporary $\mathrm{N}$ starvation on leaf photosynthetic rate and chlorophyll content of maize. Can. J. Plant Sci., 65, 491-500.

Glenn F. B., Daynard T. B., 1974. Effects of genotype, planting pattern, and plant density on plant-to-plant variability and grain yield of corn. Can. J. Plant Sci., 54, 323-330.

Goss M.-J., Ehlers W., Boone F. B., White I., Howse K. R., 1984. Effects of soil management practice on soil physical conditions affecting root growth. J. agric. Eng. Res., 30, 131-140.

Gounot M., Atry M., N'Kandza J., Yu O., 1980. Photosynthèse nette et rythme d'apparition des feuilles chez le dactyle (Dacty/is glomerata L.). C. R. Acad. Sci., Paris, 290 D, 1257-1260.

Grimes D. W., Miller R. J., Wiley P. L., 1975. Cotton and corn root development in two field soils of different strength characteris tics. Agron. J., 67, 519-523.

Grobbelaar W. P., 1963. Responses of young maize plants 10 root temperatures. Meded. Landbouwhogesch. Wageningen, 63 (5), 1-71.

Hackete C., 1969. A study of the root system of barley. II. Relationships between root dimensions and nutrient uptake. New Phytol., 68, 123-130.
Hackett C., Rose D. A., 1971a. A model of the extension and branching of a seminal root of barley and its use in studying relations between root dimensions. I. The model. Aust. J. biol. Sci., 25, 669679.

Hackett C., Rose D. A., 1971b. A model of the extension and branching of a seminal root of barley and its use in studying relation between root dimensions. II. Results and inferences from manipulation of the model. Aust. J. biol. Sci., 25, 681-690.

Hackett C., 1973. A growth analysis of the young sorghum root system. Aust. J. biol. Sci., 26, 1211-1214.

Hagin J., 1952. Influence of soil aggregation on plant growth. Soil Sci., 74, 471-478.

Harley J. I.., Scott-Russel R., 1979. In The soil root interface. New Phytol., Academic Press. London, 448 p.

Huck M. G., Klepper B., Taylor H. M., 1970. Diurnal variations in root diameter. Plant Physiol., 45, 529-530.

Huck M. G., Hillel D., 1983. A model of root growth and water uptake accounting for photosynthesis, respiration, transpiration and soil hydraulics. Adv. Irrig., 2, 273-333.

De Jager A., 1982. Effects of a localized supply of $\mathrm{H}_{2} \mathrm{PO}_{4}, \mathrm{NO}_{3}$, $\mathrm{SO}_{4}, \mathrm{Ca}$ and $\mathrm{K}$ on the production and distribution of dry matter in young maize plants. Neth. J. agric. Sci., 30, 193-203.

Jordan M.-O., 1987. Mise en place du système racinaire du mais. I. Importance de quelques paramètres relatifs à la plante. Agronomie, 7 (6), 365-371.

Kahn F., 1977. Analyse structurale des systèmes racinaires des plantes ligneuses de la forêt tropicale dense humide. Coden, CNDLAR, 32 (2), 321-358.

Kahn F., 1978. Architecture et dynamique spatiale racinaires. Symposium sur la physiologie des racines et symbioses. C. $R$., Nancy, Fra, Seichamps, 242-267.

Kaspar T. C., Woolley D. G., Taylor H. M., 1981. Temperature effect on the inclination of lateral roots of soybeans. Agron. J., 73 (2), 383-384.

Kawata S., Maruyama S., Soejima M., 1977a. Root formation in rice plant and levels of nitrogen supply. Jpn J. Crop Sci., 46 (2), 193-198.

Kawata S., Soejima M., Tabuki R., 1977b. Effect of top dressing of nitrogen fertilizer on the formation of superficial roots of rice plants. Jpn J. Crop Sci., 46 (2), 254-260.

Kiesselbach T. A., 1949. The structure and reproduction of corn. University of Nebraska Press., Reed. 1980, 96 p.

Klepper B., Rickmann R. W., Taylor H. M., 1983. Farm management and the function of field crop root systems. Agric. Water Manage., 7, 115-141.

Klepper B., Belford R. K., Rickmann R. W., 1984. Root and shoot development in winter wheat. Agron. J., 76 (1), 117-123.

Kopke U., 1981. A comparison of methods for measuring root growth of field crops. Z. Acker Pflanzenbau, 150, 39-49.

Kopke U., Bohm W., Jachmann Th., 1982. Rooting patterns of three winter wheat cultivars in a field and greenhouse experiment. Z. Acker Pflanzenbau, 151, 42-48.

Lambert J. R., Bakes D. N., 1979. Simulation of soil plants interactions. In The soil root interface. HARI Ir J. L. and SCOTT-RusSEL, New. Phytol. Academic Press. New York, 448 p.

Lambers H., Posthumus F., 1980. The effect of light intensity and relative humidity on growth and root respiration of Plantago lanceolata and Zea mays. J. exp. Bot., 31, 1621-1630.

Lambers H., 1983. "The functional equilibrum", nibbling on the edges of a paradigm. Neth. J. agric. Sci., 31 (4), 305-311.

Lemaire F., 1975. Action comparée de l'alimentation azotée sur la croissance du système racinaire et des parties aériennes des végétaux. Ann. agron., 26 (1), 59-74.

Lesaint C., Grandjean M., Coic Y., 1979. Comparaison de la composition minérale des petites et grosses racines d'une même plante (maïs et tomates). Déductions physiologiques et agronomiques. C. R. Acad. Agric. Fr., 65, 970-981.

Linscott D. L., Fox R. L., Lipps R. C., 1962. Corn root distribution and moisture extraction in relation to nitrogen fertilization and soil properties. Agron. J., 54 (3), 185-189.

Lungley D. R., 1973. The growth of root systems. A numerical computer simulation model. Plant Soil, 38, 145-159. 
Maertens C., 1964. La résistance mécanique des sols à la pénétration : ses facteurs et son influence sur l'enracinement. Ann. agron., 15, 539-554.

Maertens C., Clauzel Y., 1982. Premières observations sur l'utilisation de l'endoscopie dans l'étude de l'enracinement " in situ » des plantes cultivées (Sorghum vulgare et Lolium multiflorum). Agronomie, 2, 677-680.

Maertens C., Picard D., 1984. Quelques perspectives en matière de recherche sur l'observation « in situ 》 de l'enracinement des plantes. Poster Coll. Gerdat, Dakar, 24-27/09/1984.

Maizlich N. A., Fritton D. P., Kendall W. A., 1980. Root morphology and early development of maize at varying levels of nitrogen. Agron. J., 72 (1), 25-31.

Masle-Beynard J., Sebillotte M., 1981. Etude de l'hétérogénéité d'un peuplement de blé d'hiver. 2. Origine des différentes catégories d'individus du peuplement ; éléments de description de sa structure. Agronomie, 1 (3), 217-224.

May L. H., Chapman F. H., Aspinal D., 1966. Quantitative studies of root development. I. The influence of nutrient concentration. Aust. J. biol. Sci., 18, 25-35.

Mayaki W. C., Stone L. R., Teare I. D., 1976. Irrigated and nonirrigated soybean, corn and grain sorghum root system. Agron. J., 68, 532-534.

Miller E. C., 1916. Comparative study of the root systems and leaf areas of corn and the sorghums. J. agric. Res., 6 (9), 311-331.

Mirreh H. F., Ketcheson J. W., 1972. Influence of soil bulk density and matric pressure on soil resistance to penetration. Can. J. Soil Sci., 52, 477-483.

Mirreh H. F., Ketcheson J. W., 1973. Influence of soil water matric potential and resistance to penetration on corn root elongation. Can. J. Soil Sci., 53, 383-388.

Mosher P. N., Miller M. H., 1972. Influence of soil temperature on the geotropic response of corn roots (Zea mays L.). Agron. J., 64, 459-462.

Narda N. K., Curry R. B., 1981. Soyroot : a dynamic model of soybean root growth and water uptake. Trans. of $A S A E, 24,651-662$.

Nye P. H., Tinker P. B., 1977. Solute movement in the soil root system. Studies in ecology. Blackwell Scientific Publications. Oxford, $342 \mathrm{p}$.

Onderdonk J. J., Ketcheson J. W., 1973. Effect of soil temperature on direction of corn root growth. Plant Soil, 39, 177-186.

Parrish J. A. D., Bazzaz F. A., 1976. Underground niche separation in successional plants. Ecology, 57, 1281-1288.

Pavlychenko T. K., 1937. Quantitative study of the entire root systems of weed and crop plants under field conditions. Ecology, 18 (1), 62-79

Penman H. L., 1963. Vegetation and hydrology. Tech. Com. $n^{\circ} 55$. Commonwealth Agricultural Bureau ; Harpenden, 124 p.

Penning de Vries F. W. T., Brunsting A. H. M., van Laar H. H. 1974. Products, requirements and efficiency of biosynthesis. A quan. titative approch. J. Theor. Biol., 45, 339-377.

Petterson S., 1975. Effects of ionic strength of nutrient solutions on phosphate uptake by sunflower. Physiol. Plant., 33, 224-228.

Petterson C. M., Klepper B., Pumphrey F. V., Rickmann R. W. 1984. Restricted rooting decreases tillering and growth of winter wheat. Agron. J., 76 (5), 861-863.

Philips R. E., Kirkham D., 1962. Soil compaction in the fields and root growth. Agron. J., 54, 29-34.

Picard D., 1976. Dynamique racinaire de Panicum maximum. Jacq et apport au sol de matière organique. Thèse Doct. Ing. es-Sci., Clermont-Ferrand, $275 \mathrm{p}$

Picard D., 1984. Etude racinaire et résistance à la sécheresse. Comm. Coll. «Résistance à la sécheresse en milieu intertropical. Quelles recherches pour le moyen terme?"Dakar, 24-27/09/1984, 131-143.

Picard D., Jordan M.-O., Trendel R., 1985. Rythme d'apparition des racines primaires du maïs. I. Etude pour une variété en un lieu donné. Agronomie, 5 (8), 667-676.
Pinthus M. J., 1969. Tillering and coronal root formation in some common and durum wheat varieties. Crop Sci., 9, 267-272.

Pitman M. G., 1972. Uptake and transport of ions in barley seedlings. III. Correlation between transport to the shoots and relative growth rate. Aust. J. Biol. Sci., 25, 905-919.

Prasad S., Patil B. D., Hazra C. R., 1983. An introduction to systems and models. Ecol. Model., 18, 73-83.

Pujos Ph., 1975. Mais et facteurs climatiques. Mémoire ENITA de Bordeaux, $169 \mathrm{p}$.

Raper C. D., Barber S. A., 1970a. Rooting systems of soybeans I. Differences in root morphology among varieties. Agron. J., 62, 581-584.

Raper C. D., Barber S. A., 1970b. Rooting systems of soybeans. II. Physiological effectiveness as nutrient absorption surfaces. Agron. J., 62, 585-588.

Russelle M. P., Wilhelm W. W., Olson R. A., Power J. F., 1984 Growth analysis based on degree days. Crop Sci., 24 (1), 28-32.

Schurmann J. J., Goedewaagen M. A. J., 1971. Methods for the examination of root systems and roots. Centre for agricultura publishing and documentation, Wageningen, $87 \mathrm{p}$.

Scott-Russel R., 1977. Plant root systems. Their functions and interaction with the soil. McCraw-Hill, London, $298 \mathrm{p}$.

Sebillotte M., 1974. Agronomie et Agriculture : essai d'analyse des tâches de l'agronome. Cah. ORSTOM, Biol., 24, 3-25.

Sheppard S. C., Miller M. H., 1977. Temperature changes and the geotropic reaction of the radicle of Zea mays L. Plant Soil, 47, 631644

Tardieu F., 1984. Etude au champ de l'enracinement du maïs. Influence de l'état structural sur la répartition des racines. Conséquences sur l'alimentation hydrique. Thèse Docteur Ingénieur, INA-PG, $232 \mathrm{p}$.

Tennant D., 1976. Root growth of wheat. 1. Early patterns of multiplication and extension of wheat roots including effects of levels of nitrogen, phosphorus and potassium. Aust. J. agric. Res., 27, 183-196.

Thornley J., 1972. A balanced quantitative model for root : shoot ratios in vegetative plants. Ann. Bot., 36, 431-441.

Thornley J., 1976. Mathematical models in plant physiology. Academic Press, London, $318 \mathrm{p}$.

Vartanian N., 1972a. Induction par la sécheresse de racines courtes, tubérisées chez des plantes annuelles : crucifères et quelques autres familles. C. R. Acad. Sci. Paris, 274 D, 1497-1500.

Vartanian N., 1972b. Action morphogénétique du facteur hydrique sur le système racinaire du Sinapis alba L. Il. Etude histocytologique des ébauches racinaires produites par la sécheresse. Rev. gen. Bot., 79, 139-165.

Vartanian N., 1981. Some aspects of structural and functional modifications induced by drought in root systems. Plant Soil, 63 83-92.

Wang J., 1983. Growth simulation of the corn radicle root for various soil physical conditions. PhD Pennsylvania State University, $194 \mathrm{p}$.

Walker J. M., 1969. One degree increments in soil temperatures affect maize seedling behavior. Soil Sci. Soc. Am. Proc., 33 (5), 729-736.

Warrington I. J., Kanemasu E. T., 1983. Corn growth response to temperature and photoperiod. I. Seedling-emergence, tassel initiation and anthesis. Agron. J., 75 (5), 749-755

Weaver J. E., 1926. Root habits of corn or maize. Chap. IX, 180191. In "Root Development of field crops". McGraw-Hill-Book Company. New York, 180-191.

Wiegand C. L., Lemon E. R., 1958. A field study of some plantsoil relations in aeration. Soil Sci. Soc. Am. Proc., 22, 216-221.

Wiersma D., 1959. Water and its relation to soils and crops. IV. The soil environment and root development. Adv. Agron., 9, 43-51.

Zuber M., 1968. Evaluations of corn root systems under various environments. Rep. 23rd on Sorghum Conf. Chicago, 67-75. 